\title{
New Spectral Observations of the Variable Galaxy Kaz 163
}

\author{
Emilia L. Karapetyan
}

Yerevan State University, Yerevan, Armenia

\begin{abstract}
Kaz 163 is a close double galaxy. Its southern component S is compact, with a very blue nucleus, in which heated active processes take place. From time to time gas formations are ejected from it, which behave themselves like emission components around the main emission lines $\mathrm{H} \alpha$ and $\mathrm{H} \beta$, around both from their long-wave and short-wave sides. This paper presents the spectral data of new observations, which were carried out with the $2.6 \mathrm{~m}$ telescope at the Byurakan Astrophysical Observatory in September 2011. During the former observation in October 1981, lines [NII] $\lambda \lambda 6584,6548$ were not visible in the spectrum of the component S. In 2001 they were already visible on the spectrum, and on the spectrum obtained in 2011 they already surpassed the intensity of $\mathrm{H} \alpha$. The magnitude of the component $\mathrm{S}$ is also changing: its nucleus is very blue and its $\mathrm{U}-\mathrm{B}=-0^{m} .63$. In the soft X-ray spectral range $(0.1-2 \mathrm{keV})$ the flux of the radiation changed by $45 \%$ during $55,000 \mathrm{sec}$, and in the hard one $(2-10 \mathrm{keV})$ it changed up to 3.4 times. Photoindices $\Gamma$ for the soft and hard ranges in the spectrum of galaxy $S$, unlike other objects, do not so much differ from each other. The mean value for the first interval is approximately 2.5 and is equal -2.0 for the second one. On the histogram of redshifts Kaz 163 corresponds to the first big peak of the distribution. It is concluded that the component $\mathrm{S}$ of the galaxy Kaz 163 is a NLS1 galaxy, with the development of their evolution, is in the preliminary stage. Component $\mathrm{N}$ is a normal elliptical galaxy with no activity.
\end{abstract}

Two spectra of the component S of the galaxy Kaz 163 (Kazarian 1983) were obtained with the $2.6 \mathrm{~m}$ telescope at the Byurakan Astrophysical Observatory (BAO) on September 25, 2011, as well as two of the component N on March 25, 2009 with the same telescope. The spectra were recorded by means of the SCORPIO camera and a grism $\left(\mathrm{C}=600 \mathrm{~mm}^{-1}\right)$ with a central wavelength of $\lambda=5200 \AA$. This system covers the spectral range $\lambda 3920-7420 \AA$ with a dispersion of $1.7 \AA$ /pixel. The 2 " wide, 5 ' high slit of the spectral camera was oriented East-West. HD 217086 was used as a comparison star. The spectra were analyzed using the MIDAS program and the spectra are given in Fig. 1a. $\mathrm{H} \alpha, \mathrm{HeI} \lambda 5876$, [OIII] $\lambda \lambda 5007,4959, \mathrm{H} \beta, \mathrm{HeII} \lambda 4686, \mathrm{H} \gamma,[\mathrm{OIII}] \lambda 4363$ emission lines were observed for the component $\mathrm{S}$. The last two lines are merged because they are too close. Only the absorption $\mathrm{NaI} \lambda 5893$ and $\mathrm{MgI} \lambda 5184$ lines and the $\mathrm{G}$ band typical of elliptical galaxies are seen for the component $\mathrm{N}$.

Kaz163 was first presented as an UV-excess galaxy in 1978. Observations of Kaz163 have been made with many telescopes at various observatories throughout the world, as well as with space telescopes, mainly in the X-ray.

Data obtained during 1984 and 1986 showed that galaxy $\mathrm{S}$ is variable, with changes in both line and continuum intensities. Spectral observations of galaxy $\mathrm{S}$ at the $3 \mathrm{~m}$ Shane telescope at the Lick Observatory in the $\lambda \lambda 4000-7500 \AA$ range and at the $1.8-\mathrm{m}$ telescope at the Asiago Observatory on July 19, 1993, in the $\lambda \lambda 3600-7600 \AA$ range are given in Leighly (1999). There it was shown that an explosion was observed in the nucleus of galaxy S in 1981, so that over the time between May 28, 1982 and September 30, 1984, its spectral characteristics changed significantly: the equivalent widths of the emission lines decreased after 1982. The line profiles also changed strongly. After 1984 the physical 

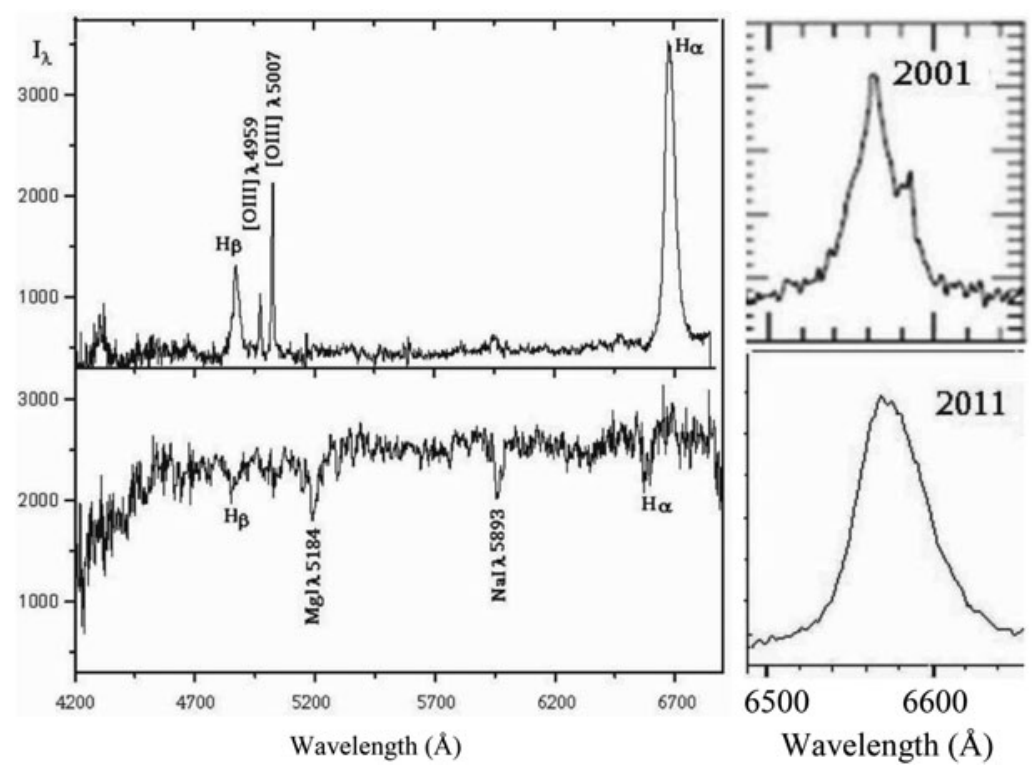

Figure 1. a) The spectra of components S (upper frame) and N (lower frame). b) Shape of $\mathrm{H} \alpha$ line observed in 2001 and 2011.

parameters and the line profiles remained essentially unchanged until 1986. During this time interval the HeII $\lambda 4686 \AA^{\circ}$ and HeI $\lambda 5876 \AA$ lines became much stronger, and remained almost unchanged from 1986 to 1989. Our observations of Kaz 163 cover a period of 30 years. In terms of spectral structure and physical characteristics, the spectra obtained over that time are quite different, as already noted above. That spectrum is shown in Fig. $1 \mathrm{~b}$, and all the collected data yields some important conclusions regarding the variability of the physical parameters of galaxy S:

1. The southern component $\mathrm{S}$ of the galaxy Kaz 163 is compact and has a very blue nucleus ( $\left.\mathrm{U}-\mathrm{B}=-0^{m} .63\right)$ in which stormy active processes are taking place. From time to time gaseous formations are ejected from it showing up as emission components on both sides of the major emission lines, $\mathrm{H} \alpha, \mathrm{H} \beta$, etc. The northern component $\mathrm{N}$ of $\mathrm{Kaz}$ 163 is a typical elliptical galaxy with no activity.

2. Over roughly 30 years the half widths, equivalent widths, and relative intensities of the emission lines have changed quite strongly.

3. In October 1981 the $[\mathrm{NII}] \lambda \lambda 6583,6548$ lines did not show up in the spectrum of galaxy S, in 2001 they were already quite noticeable, and in September 2011 their intensities already exceeded that of $\mathrm{H} \alpha$.

4. In the soft $\mathrm{x}$-ray region $(0.1-2 \mathrm{keV})$ the radiative flux varied by $45 \%$ over a time of $55,000 \mathrm{~s}$, and in the hard x-ray region $(2-10 \mathrm{keV})$ the flux changed by up to a factor of 3.4 .

5. Based on the data discussed here, we conclude that component $\mathrm{S}$ of the galaxy Kaz 163 is, by all probability, an NLS1 galaxy and, in terms of its evolutionary development, it is in a preliminary stage.

\section{References}

Kazaryan, M. A. 1983, Astrofizika 19, 411

Leighly, K. M. 1999, ApJS 125, 297 